\title{
Desenvolvimento Vegetativo de Cesalpinea ferrea e Anadenanthera macrocarpa: I - Estimativa das Temperaturas Cardinais
}

\author{
Larissa Vieira Da Silva $^{1}$ (D), Fernando Yuri da Silva Reis ${ }^{1}$ (D), Fabrina Bolzan Martins ${ }^{1}$ (D), \\ Jefferson Martiniano Cassemiro ${ }^{1}$ \\ ${ }^{1}$ Instituto de Recursos Naturais, Universidade Federal de Itajubá, Itajubá, MG, Brasil.
}

Recebido em: 28 de Dezembro de 2018 - Aceito em: 30 de Setembro de 2019

\begin{abstract}
Resumo
A temperatura do ar influencia o desenvolvimento vegetativo e a duração da fase de muda de espécies florestais, uma vez que regula as reações fotoquímicas e bioquímicas da fotossíntese, e por isso é considerada a principal variável meteorológica. O objetivo deste trabalho foi estimar as temperaturas cardinais (basal inferior, ótima e basal superior), que determinam os limiares em que ocorrem desenvolvimento vegetativo, e estimar o filocrono de duas espécies florestais na fase de muda. Para isso, foi instalado um experimento a campo, sob delineamento inteiramente casualizado, em esquema fatorial $(2 \times 12)$, sendo duas espécies florestais nativas (pau-ferro - Caesalpinia ferrea Mart. ex. Tul. Var. leiostachya Benth e angico-vermelho - Anadenanthera macrocarpa (Benth) Brenan) e doze épocas de semeadura, com cinco repetições por tratamento. Os valores das temperaturas cardinais para o pau pau-ferro foram: $12,8^{\circ} \mathrm{C}, 20,7^{\circ} \mathrm{C}$ e $46,3{ }^{\circ} \mathrm{C}$ e para o angico-vermelho foram: $10,3{ }^{\circ} \mathrm{C}, 23{ }^{\circ} \mathrm{C}$ e $45,5{ }^{\circ} \mathrm{C}$. Já o filocrono médio estimado para o pau-ferro foi de $56,9^{\circ} \mathrm{C}$ dia folha ${ }^{-1}$ e de $163,8{ }^{\circ} \mathrm{C}$ dia folha ${ }^{-1}$ para o angico-vermelho, mostrando que o angico-vermelho necessita de mais energia para promover o desenvolvimento vegetativo quando comparado ao pau-ferro.
\end{abstract}

Palavras-chave: temperatura basal inferior, temperatura ótima, temperatura basal superior.

\section{Vegetative Development of Cesalpinea ferrea and Anadenanthera macrocarpa: I: Estimated of Cardinal Temperatures}

\begin{abstract}
The air temperature influences the vegetative development and the seedling phase duration in the forest species, since it regulates the photosynthesis reactions, because that is considered the main meteorological variable. The aim of this work was to estimate cardinal temperatures (base, optimum and maximum), which determines the thresholds on the development phases, and to estimate the phyllochron of two forest species in the seedling phase. A field experiment was carried out in a completely randomized design, in a 2X12 factorial arrangement, with two Brazilian forest species (Caesalpinia ferrea Mart. ex. Tul. Var. leiostachya Benth e angico-vermelho - Anadenanthera macrocarpa (Benth) Brenan), 12 sowing dates and five replicates. The cardinal temperatures for pau-ferro were: $12.8^{\circ} \mathrm{C}, 20.7{ }^{\circ} \mathrm{C}$ and $46.3{ }^{\circ} \mathrm{C}$ and for angico-vermelho were: $10.3{ }^{\circ} \mathrm{C}, 23{ }^{\circ} \mathrm{C}$ and $45.5{ }^{\circ} \mathrm{C}$. The phyllochron was $56.9{ }^{\circ} \mathrm{C}$ day per leaf for pau-ferro and was $163.8^{\circ} \mathrm{C}$ day per leaf for angico-vermelho, which indicates that angico-vermelho requires more energy accumulation to promote the vegetative development than pau-ferro.
\end{abstract}

Keywords: base temperature, optimum temperature and maximum temperature.

\section{Introdução}

Os processos fisiológicos e metabólicos das espécies florestais são dependentes da temperatura do ar, uma vez que regula as reações fotoquímicas e bioquímicas da fotossíntese (Ruelland e Zachowski, 2010; Taiz et al., 2018), influenciando também o transporte e translocação de solutos e o balanço entre a transpiração e o consumo de água no solo (Callejas et al., 2014; Freitas et al., 2017).

Autor para correspondência: Fabrina Bolzan Martins, fabrinabm@gmail.com. 
Por sua vez, estes processos refletem no desenvolvimento vegetativo (DV), como a germinação (Bahuguna e Jagadish, 2015) e a taxa de emissão de folhas (Erpen et al., 2013; Souza e Martins, 2014), e no desenvolvimento reprodutivo (DR) como florescimento, frutificação e maturação dos frutos (García - Mozo et al., 2010; Martins et al., 2019).

Neste caso, a metodologia mais usual para descrever o efeito da temperatura do ar é através do filocrono $\left({ }^{\circ} \mathrm{C}\right.$ dia folha $^{-1}$ ) que representa o acúmulo térmico necessário para o DV e DR (Freitas et al., 2017; Ferreira et al., 2019) delimitado pelas temperaturas cardinais, denominadas temperatura basal inferior (Tb), ótima (Tot) e basal superior (TB) (Arnold, 1959; Sinclair et al., 2004; Freitas et al., 2017). As Tb e TB representam, respectivamente, as temperaturas abaixo e acima das quais os processos fisiológicos e metabólicos são reduzidos (Yang et al., 1995; Ruelland e Zachowski, 2010), limiar em que DV e DR é nulo ou desprezível para fins de cálculo (Souza e Martins, 2014). Valores de temperatura próximos à $\mathrm{Tb}$ reduzem todas as reações metabólicas da fotossíntese, enquanto que $\sim$ TB tendem a elevar os custos de respiração de manutenção, processo que consome energia ou esqueletos carbonados provenientes da fotossíntese, por aumentar a renovação de proteínas e a manutenção do balanço de íons (Marenco e Lopes, 2009; Ruelland e Zachowski , 2010). Já a Tot representa a temperatura em que ocorre o máximo DV e DR.

Os valores de $\mathrm{Tb}$, Tot e TB variam entre espécies, cultivares e variedades e também em função dos subperíodos de DV e DR (Lago et al., 2009; Souza e Martins, 2014; Martins et al., 2019). Podem ser estimados através de métodos diferenciados (Yang et al., 1995; Freitas et al., 2017; Ferreira et al., 2019), os quais utilizam em comum dados de observações fenológicas e de temperatura do ar (Lago et al., 2009; Souza e Martins, 2014). Para a estimativa $\mathrm{Tb}$ convenciona-se utilizar o método do menor valor do quadrado médio do erro (QME) pela sua praticidade e simplicidade (Sinclair et al., 2004; De Paula e Streck, 2008; Freitas et al., 2017). No QME é necessário que as plantas sejam expostas a condições de temperaturas amenas, as quais ocorrem em Minas Gerais durante os meses de outono e inverno (Garcia et al., 2018). Para a estimativa da Tot, as plantas são submetidas às condições ideais de desenvolvimento, que geralmente são obtidas somente em câmaras de crescimento (Soltani e Sinclair, 2012; Muttoni et al., 2017), dificultando a praticidade da estimativa da Tot, exceto somente na fase do DV entre a semeaduraemergência (Muttoni et al., 2017), e por esse motivo, têmse utilizado para esse fim a metodologia proposta por Lisboa et al., (2012). Quanto a TB, geralmente seus valores são elevados e não são atingidos no campo, ainda assim, para a sua estimativa é necessário que as plantas sejam expostas a temperaturas mais elevadas em que ocorra menor DV e DR (Freitas et al., 2017).
O conhecimento das temperaturas cardinais e do filocrono é fundamental para o sucesso dos programas de reflorestamento e recuperação de áreas degradadas, pois permite a escolha de espécies mais adaptadas às condições climáticas do local de cultivo, a produção de mudas de melhor qualidade, garantindo o estabelecimento das mesmas no campo (Ferreira et al., 2019). Além disso, são valores utilizados em modelos de DV e DR e em modelos de simulação dos impactos das mudanças climáticas (Streck et al., 2013). Encontram-se na literatura diversos estudos dessa natureza em culturas anuais e perenes de interesse comercial (Sinclair et al., 2004; Lima e Silva, 2008; Lago et al., 2009; Streck et al., 2009; Lucas et al., 2012; Erpen et al., 2013; Erpen et al., 2013; Callejas et al., 2014; Freitas et al., 2017; Martins et al., 2019). Entretanto, há poucos estudos para culturas perenes, principalmente em espécies nativas de menor interesse comercial (Fagundes et al., 2010; Ferreira et al., 2019).

Nesse sentido, o pau-ferro (Caesalpinia ferrea Mart. ex. Tul. Var. leiostachya Benth) e o angico-vermelho (Anadenanthera macrocarpa (Benth) Brenan) são duas espécies florestais nativas que apresentam potencialidade de estudo, visto que possuem usos múltiplos, apresentam durabilidade natural, ampla dispersão, boa regeneração, elevada adaptabilidade, rápido crescimento, sendo preferidas na produção de mudas em viveiros florestais (Biruel et al., 2010; Baldin et al., 2015; Dias et al., 2012). Portanto, o presente trabalho teve como objetivo estimar as temperaturas cardinais ( $\mathrm{Tb}$, Tot e $\mathrm{TB}$ ) e o filocrono para o DV do pau-ferro e angico-vermelho durante a fase de muda.

\section{Material e Métodos}

O experimento foi conduzido a campo na área experimental da Universidade Federal de Itajubá (22 ${ }^{\circ}$ 24'46.43" S; 45²6'48.94" O; 850 m de altitude), em Itajubá (MG), durante os anos agrícolas de 2017 e 2018. Segundo a classificação de Köppen, o clima é Cwa, tropical de altitude, caracterizado verões quentes e chuvosos, e invernos secos (Martins et al., 2018).

$\mathrm{O}$ delineamento utilizado foi o inteiramente casualizado, organizado em esquema fatorial ( $2 \times 12)$, sendo duas espécies florestais: pau-ferro (Caesalpinia ferrea) e angico-vermelho (Anadenanthera macrocarpa), doze épocas de semeadura (ES), com cinco repetições por tratamento, totalizado 120 unidades experimentais (UE). As ES foram instaladas a cada 30 dias, a fim de que as plantas fossem expostas a diferentes condições meteorológicas durante seu desenvolvimento, conforme recomendações de Freitas et al. (2017). A semeadura foi realizada através do método direto e após as UE atingirem 50\% de emergência (Tabela 1), realizou-se o raleio, deixando duas mudas por UE. 
Tabela 1 - Datas de semeadura, emergência e duração da fase de muda para cada época e espécies pau-ferro e angico-vermelho. Itajubá (MG), $2017 / 2018$.

\begin{tabular}{|c|c|c|c|c|c|}
\hline \multirow[t]{2}{*}{ Épocas de semeadura } & \multirow[t]{2}{*}{ Datas de semeadura } & \multicolumn{2}{|c|}{ pau-ferro } & \multicolumn{2}{|c|}{ angico-vermelho } \\
\hline & & Emergência* & Duração** & Emergência* & Duração** \\
\hline ES1 & $20 / 01 / 2017$ & $10 / 02 / 2017$ & 119 & $10 / 02 / 2017$ & 84 \\
\hline ES2 & $20 / 02 / 2017$ & 03/03/2017 & 168 & 03/03/2017 & 245 \\
\hline ES3 & $20 / 03 / 2017$ & $31 / 03 / 2017$ & 231 & $31 / 03 / 2017$ & 266 \\
\hline ES4 & $20 / 04 / 2017$ & 05/05/2017 & 168 & $28 / 04 / 2017$ & 259 \\
\hline ES5 & $19 / 05 / 2017$ & $02 / 06 / 2017$ & 154 & 02/06/2017 & 252 \\
\hline ES6 & $20 / 06 / 2017$ & $14 / 07 / 2017$ & 154 & 07/07/2017 & 238 \\
\hline ES7 & $20 / 07 / 2017$ & $18 / 08 / 2017$ & 105 & $04 / 08 / 2017$ & 196 \\
\hline ES8 & $21 / 08 / 2017$ & $15 / 09 / 2017$ & 112 & 01/09/2017 & 112 \\
\hline ES9 & 20/09/2017 & $13 / 10 / 2017$ & 161 & 06/10/2017 & 84 \\
\hline ES10 & $20 / 10 / 2017$ & $10 / 11 / 2017$ & 70 & 03/11/2017 & 105 \\
\hline ES11 & $20 / 11 / 2017$ & $08 / 12 / 2017$ & 77 & $15 / 12 / 2017$ & 84 \\
\hline ES12 & $20 / 12 / 2017$ & $19 / 01 / 2018$ & 77 & $19 / 01 / 2018$ & 63 \\
\hline
\end{tabular}

*Emergência foi considerada o dia em que se contabiliza 50\% de germinação, que delimita o início (i) de cada época de semeadura; ** Período em dias que se estende desde a emergência até quando havia emitido, em média, 20 (pau-ferro) e 12 (angico-vermelho) folhas.

Cada UE foi composta por vaso de polietileno de 11 litros ( $23 \mathrm{~cm}$ de altura, $24 \mathrm{~cm}$ de diâmetro superior e 20 diâmetro inferior), com as paredes externas e internas pintadas de branco, para evitar o aumento da temperatura do substrato pela absorção de radiação solar. Os vasos foram preenchidos com o horizonte A moderado de um Latossolo Vermelho distrófico típico, coletado em Itajubá. Após analises laboratoriais verificou-se que possuía acidez elevada ( $\mathrm{pH}$ em $\left.\mathrm{H}_{2} \mathrm{O}=5\right)$, baixa quantidade de cálcio e magnésio trocáveis (respectivamente 0,16 e $0 \mathrm{cmol}_{\mathrm{c}} / \mathrm{dm}^{3}$ ), baixos teores de fósforo, potássio, cálcio e magnésio, enquanto os teores de cobre, ferro, manganês e zinco foram considerados altos. As considerações e as correções do solo seguiram a recomendação proposta pelo Guimarães et al. (1999), em que foi aplicado em cada UE: para correção da acidez e diminuição do teor de $\mathrm{Al}^{3+}: 17,63$ gramas de carbonato de cálcio e 5,88 gramas de carbonato de magnésio; para a correção das deficiências nutricionais relacionadas aos macronutrientes: 8,86 gramas de superfosfato simples (18\%), 1,52 gramas de cloreto de potássio $(60 \%)$ e 2,28 gramas sulfato de amônia.

O DV foi quantificado através do número de folhas emitidas na haste principal (NF) durante a fase de muda (FM). A FM para o pau-ferro foi definida pela contabilização do NF desde a emergência até, em média, $20 \mathrm{NF}$ (Martins et al., 2007; Abreu et al., 2015). Para o angicovermelho foi utilizado o patamar de $12 \mathrm{NF}$, o qual é o ideal para a comercialização de mudas desta espécie e reflete, em média, $40 \mathrm{~cm}$ de altura (Oliveira et al., 2016).

Os dados de temperatura do ar foram obtidos através de uma estação meteorológica automática, instalada na área experimental. Pela recomendação de Freitas et al., (2017) e Ferreira et al., (2019) foram utilizadas para a estimativa da Tb as ES que possuem os menores valores de temperatura do ar e ao mesmo tempo maior duração da
FM, para a TB foram utilizadas as duas ES com maiores valores de Tmed e ao mesmo tempo maior duração da FM. Já para a estimativa da Tot foram utilizadas as doze ES.

\subsection{Estimativa da temperatura basal inferior $(\mathrm{Tb})$}

Para a estimativa da Tb utilizou o método do QME (Sinclair et al., 2004). Para cada espécie e cada ES foram ajustadas regressões lineares simples entre o NF e o grausdia acumulados $\left(\mathrm{GD},{ }^{\circ} \mathrm{C}\right.$ dia), dado por:

$$
\begin{aligned}
& \mathrm{GD}=\sum_{\mathrm{i}=1}^{\mathrm{n}}(\mathrm{Tmed}-\mathrm{Tb}), \\
& \text { se } \text { Tmed } \leq \mathrm{Tb} \text {, então } \text { Tmed }=\mathrm{Tb}
\end{aligned}
$$

em que Tmed = temperatura média diária do ar, obtida pela média aritmética das temperaturas horárias da estação meteorológica automática $\left({ }^{\circ} \mathrm{C}\right) ; \mathrm{Tb}=$ temperatura basal inferior $\left({ }^{\circ} \mathrm{C}\right) ; \mathrm{i}=$ data de emergência (Tabela 1 ); e $\mathrm{n}$ é a data do término da fase de muda (Tabela 1). Para o cálculo do GD, utilizou-se uma série de Tb's que variavam de 0 a $20{ }^{\circ} \mathrm{C}$, a intervalos de $0,5^{\circ} \mathrm{C}$ (Lucas et al., 2012; Sinclair et al., 2004).

$\mathrm{O}$ valor da $\mathrm{Tb}$, para cada época, foi aquele que apresentou o menor valor de QME das regressões lineares, enquanto o valor da $\mathrm{Tb}$, para cada espécie, foi obtido pela média aritmética dos valores de $\mathrm{Tb}$ encontrados nas diferentes épocas (Freitas et al., 2017).

\subsection{Estimativa da temperatura ótima (Tot)}

Para a estimativa da Tot utilizou o método proposto por Lisboa et al. (2012), baseado na média da temperatura do ar daquelas ES que apresentam maior DV. Para isso, primeiramente determinou-se, para cada espécie, ES e U.E, o filocrono, dado pelo inverso do coeficiente angular (a) da regressão linear entre o NF e o GD a partir da data 
de emergência (De Paula e Streck, 2008; Freitas et al., 2017) (Eq. 2):

$$
\mathrm{NF}=\mathrm{a} \cdot \mathrm{GD}+\mathrm{b}
$$

No cálculo do GD foi considerado o valor de Tb estimado pelo QME.

Posteriormente, foi realizada a verificação de normalidade dos dados de filocrono através do teste de Shapiro-Wilk $(\alpha=0,05)$. Os dados que não seguiram a normalidade foram transformados em $\mathrm{Ln}(\mathrm{x})$ (Freitas et al., 2017). Os valores do filocrono foram submetidos à análise de variância (ANOVA), para avaliar o efeito das fontes de variação (espécies e ES) e as médias foram comparadas pelo teste de Scott-Knott, $1974(\alpha=0,05)$. Com esses passos foi possível detectar a ES que apresenta menor filocrono e indica o maior DV (Martins et al., 2007; Lisboa et al., 2012; Freitas et al., 2017). Todos os testes foram realizados no software SISVAR 5.6 (Ferreira, 2014).

\subsection{Estimativa da temperatura basal superior (TB)}

Para a estimativa da TB foi utilizada a metodologia proposta por Ometto (1981), modificada por Lima e Silva (2008) e utilizada por Freitas et al. (2017). Neste método o valor de TB é fornecido indiretamente através de dois casos (I e II):

$$
\begin{aligned}
& \text { Caso I: } \mathrm{TB}>\mathrm{Tmed}>\mathrm{Tb} \\
& \mathrm{A}=\mathrm{N}_{2} \cdot \mathrm{TM}_{1} \cdot \mathrm{TM}_{2}-\mathrm{N}_{2} \cdot \mathrm{TM}_{2} \cdot \mathrm{Tm}_{1} \\
& \mathrm{~B}=-\mathrm{N}_{1} \cdot \mathrm{TM}_{1} \cdot \mathrm{TM}_{2}+\mathrm{N}_{1} \cdot \mathrm{TM}_{1} \cdot \mathrm{Tm}_{2} \\
& \mathrm{C}=\left(-\mathrm{TM}_{1}+\mathrm{Tm}_{1}\right) \cdot\left(-\mathrm{TM}_{2}+\mathrm{Tm}_{2}\right) \\
& \mathrm{D}=\mathrm{N}_{1} \cdot \mathrm{Tm}_{2}{ }^{2} \cdot \mathrm{N}_{2}-2 \cdot \mathrm{Tm}_{2} \cdot \mathrm{N}_{1} \cdot \mathrm{N}_{2} \cdot \mathrm{Tb}-\mathrm{Tm}_{2} \cdot \mathrm{Tm}_{1} \cdot \mathrm{N}_{1}{ }^{2} \\
& \mathrm{E}=-T m_{2} \cdot \mathrm{TM}_{1} \cdot \mathrm{N}_{1}{ }^{2}+\mathrm{N}_{2}{ }^{2} \cdot \mathrm{TM}_{1} \cdot \mathrm{Tm}_{2} \\
& -\mathrm{Tm}_{1} \cdot \mathrm{N}_{2}{ }^{2} \cdot \mathrm{Tm}_{2}+2 \cdot \mathrm{Tm}_{2} \cdot \mathrm{N}_{2}{ }^{2} \cdot \mathrm{Tb} \\
& \mathrm{F}=\mathrm{Tm}_{1} \cdot \mathrm{N}_{1}{ }^{2} \cdot \mathrm{TM}_{2}-2 \cdot \mathrm{N}_{2}{ }^{2} \cdot \mathrm{TM}_{1} \cdot \mathrm{Tb}-2 \cdot \mathrm{N}_{1}{ }^{2} \cdot \mathrm{TM}_{2} \cdot \mathrm{Tb} \\
& \mathrm{G}=\mathrm{TM}_{1} \cdot \mathrm{N}_{1}{ }^{2} \cdot \mathrm{TM}_{2} \\
& +\mathrm{Tm}_{1}^{2} \cdot \mathrm{N}_{2} \cdot \mathrm{N}_{1}-2 \cdot \mathrm{Tm}_{1} \cdot \mathrm{N}_{2} \cdot \mathrm{N}_{1} \cdot \mathrm{Tb} \\
& \mathrm{H}=2 \cdot \mathrm{Tm}_{1} \cdot \mathrm{N}_{2}^{2} \cdot \mathrm{Tb}+2 \cdot \mathrm{N}_{1} \cdot \mathrm{TM}_{2} \\
& \cdot \mathrm{N}_{2} \cdot \mathrm{Tb}+2 \cdot \mathrm{N}_{2} \cdot \mathrm{TM}_{1} \cdot \mathrm{N}_{1} \cdot \mathrm{Tb} \\
& \mathrm{I}=-2 \cdot \mathrm{N}_{2} \cdot \mathrm{TM}_{1} \cdot \mathrm{N}_{1} \cdot \mathrm{TM}_{2} \\
& +\mathrm{N}_{2}{ }^{2} \cdot \mathrm{TM}_{1} \cdot \mathrm{TM}_{2}-\mathrm{Tm} 1 \cdot \mathrm{N}_{2}{ }^{2} \cdot \mathrm{TM}_{2} \\
& \mathrm{~J}=-\mathrm{N}_{1} \cdot \mathrm{TM}_{2}+\mathrm{N}_{1} \cdot \mathrm{Tm}_{2}+\mathrm{N}_{2} \cdot \mathrm{TM}_{1}-\mathrm{N}_{2} \cdot \mathrm{Tm}_{1} \\
& \mathrm{~TB}=\frac{\mathrm{A}+\mathrm{B} \pm \sqrt{\mathrm{C} \cdot(\mathrm{D}+\mathrm{E}+\mathrm{F}+\mathrm{G}+\mathrm{H}+\mathrm{I})}}{\mathrm{J}}
\end{aligned}
$$

Em que: $\mathrm{N}_{1}$ e $\mathrm{N}_{2}=$ número de dias de duração da fase de muda; $\mathrm{TM}_{1}$ e $\mathrm{TM}_{2}=$ temperaturas máximas do ar $\left({ }^{\circ} \mathrm{C}\right)$; $\mathrm{Tm}_{1}$ e $\mathrm{Tm}_{2}=$ temperaturas mínimas do ar $\left({ }^{\circ} \mathrm{C}\right) ; 1$ e $2=$ épocas de semeadura com maiores valores de temperatura média do ar.

$$
\begin{aligned}
& \text { Caso II: } \mathrm{TB}>\mathrm{Tb}>\text { Tmed } \\
& \mathrm{A}=-\mathrm{N}_{1} \cdot \mathrm{Tb} \cdot \mathrm{TM}_{2}+\mathrm{N}_{1} \cdot \mathrm{Tb} \cdot \mathrm{Tm}_{2}+\mathrm{N}_{2} \cdot \mathrm{TM}_{1} \cdot \mathrm{Tb} \\
& \mathrm{B}=-\mathrm{N}_{2} \cdot \mathrm{Tm}_{1} \cdot \mathrm{Tb}-2 \cdot \mathrm{N}_{2} \cdot \mathrm{TM}_{1} \cdot \mathrm{TM}_{2} \\
& +2 \cdot \mathrm{N}_{2} \cdot \mathrm{TM}_{2} \cdot \mathrm{Tm}_{1} \\
& \mathrm{C}=2 \cdot \mathrm{N}_{1} \cdot \mathrm{TM}_{1} \cdot \mathrm{TM}_{2}-2 \cdot \mathrm{N}_{1} \cdot \mathrm{TM}_{1} \cdot \mathrm{Tm}_{2} \\
& \mathrm{D}=\mathrm{N}_{1} \cdot \mathrm{TM}_{2}-\mathrm{N}_{1} \cdot \mathrm{Tm}_{2}-\mathrm{N}_{2} \cdot \mathrm{TM}_{1}+\mathrm{N}_{2} \cdot \mathrm{Tm}_{1} \\
& \mathrm{~TB}=\frac{(\mathrm{A}+\mathrm{B}+\mathrm{C})}{\mathrm{D}}
\end{aligned}
$$

Em que: $\mathrm{N}_{1}$ e $\mathrm{N}_{2}$ são as durações de cada época de semeadura; $\mathrm{TM}_{1}$ e $\mathrm{TM}_{2}$ são as temperaturas máximas das duas épocas e $\mathrm{Tm}_{1}$ e $\mathrm{Tm}_{2}$, as temperaturas mínimas das épocas de semeadura. 1 e 2 = épocas de semeadura com maiores valores de temperatura média do ar.

\section{Resultados e Discussão}

Durante o experimento as duas espécies foram submetidas a significativas variações de temperatura do ar (Figura 1). Os valores oscilaram entre $34,6{ }^{\circ} \mathrm{C}$, máximo absoluto (observados durante ES3, ES4, ES5, ES6, ES7, ES8), e $3,6^{\circ} \mathrm{C}$, mínimo absoluto (observado nas ES2, ES3, ES4, ES5, ES6), o que é necessário neste tipo de estudo. Tais variações permitem que a planta se desenvolva de forma diferenciada, permitindo confiabilidade na estimativa das temperaturas cardinais (Souza e Martins, 2014; Freitas et al., 2017; Ferreira et al., 2019). Adicionalmente, os valores de Tmed obtido entre as ES $\left(17,9^{\circ} \mathrm{C}\right.$ $23,5^{\circ} \mathrm{C}$ ) estão em acordo com a climatologia de Itajubá (entre $\sim 17{ }^{\circ} \mathrm{C}$ e $\sim 24{ }^{\circ} \mathrm{C}$ ), assim como a Tmax (entre $\sim 25^{\circ} \mathrm{C}$ e $\sim 30{ }^{\circ} \mathrm{C}$ ) e Tmin (entre $\sim 10{ }^{\circ} \mathrm{C}$ e $\sim 15^{\circ} \mathrm{C}$ ) (Garcia et al., 2018).

A exposição a diferentes condições meteorológicas durante as ES influenciou a duração da FM, onde se observou uma relação inversa entre temperatura do ar e duração da FM, mesma tendência constatada em estudos com espécies perenes, como Souza e Martins (2014) para as cultivares de oliveira Grappolo e MGS Mariense, Ferreira et al. (2019) para o pau viola (Citharexylum myrianthum Cham.) e urucum (Bixa orellana L.), e Freitas et al. (2017) para duas espécies de eucalipto.

Para o pau-ferro, as ES2, ES3, ES4 e ES5 apresentaram maior duração da FM e menores valores de temperaturas do ar, sendo as épocas escolhidas para estimativa da Tb. Já para o angico-vermelho, além das ES2, ES3, ES4 e ES5, considerou-se também a ES6, uma vez que apresentou grande duração da FM (238 dias). Por outro lado, as épocas ES8 e ES9, para o pau-ferro, e ES7 e ES8 para o angico-vermelho apresentaram simultaneamente elevada duração da FM em condições de alta temperatura média do ar, sendo as duas ES escolhidas para a estimativa da TB, seguindo as recomendações de Freitas et al. (2017) e Ferreira et al. (2019). 
$\mathrm{Na}$ estimativa da $\mathrm{Tb}$, todas as regressões entre o NF e GD para ambas as espécies tiveram valores de QME baixos (menores que 0,9785 para o pau-ferro e 0,3992 para o angico-vermelho) (Figura 2), coeficiente de ajuste $\left(R^{2}\right)$ elevados $(\geq 0,96$ para ambas espécies) (Figuras $3 \mathrm{e}$ $4)$, e $\alpha$ significativos $(\mathrm{p} \leq 0,05)$, sendo características desejáveis à estimativa da $\mathrm{Tb}$ (Lucas et al., 2012; Martins et al., 2012; Freitas et al., 2017).

Houve pequena variação entre os valores de Tb estimados para o pau-ferro (Figura 2 A). Na ES2, o menor valor que QME $(0,4182)$ foi para a $\mathrm{Tb}$ de $11{ }^{\circ} \mathrm{C}$, na ES3 $(0,1441)$ foi para a Tb de $14{ }^{\circ} \mathrm{C}$, na ES4 $(0,8684)$ foi para a $\mathrm{Tb}$ de $13,5^{\circ} \mathrm{C}$ na $\operatorname{ES} 5(0,9785)$ foi para a Tb de $12,5^{\circ} \mathrm{C}$. Já para o angico-vermelho (Figura $2 \mathrm{~B}$ ) houve grande variação entre os valores de $\mathrm{Tb}$ estimados, mesma tendência observada por Lucas et al. (2012) e Freitas et al. (2017). Nas ES2 $(\mathrm{QME}=0,3285)$ e ES5 $(\mathrm{QME}=0,3992)$ os menores valores de $\mathrm{QME}$ corresponderam a de $0{ }^{\circ} \mathrm{C}$, o que não é considerado realístico (Lago et al., 2009), sendo desconsideradas no valor final da $\mathrm{Tb}$ do angico-vermelho. Ainda para o angico-vermelho, os menores valores de QME nas ES3(QME $=0,1370)$, ES4 $(\mathrm{QME}=0,2474) \mathrm{e}$
ES6 (QME $=0,2774$ ) foram encontrados para as Tb's de $11,5{ }^{\circ} \mathrm{C}, 13{ }^{\circ} \mathrm{C}$ e $6,5{ }^{\circ} \mathrm{C}$, respectivamente. Deste modo, o valor final da $\mathrm{Tb}$ para o pau-ferro foi de $12,8^{\circ} \mathrm{C}$, os quais foram obtidos pelas médias aritméticas das ES2, ES3, ES4 e ES5; e do angico-vermelho foi de $10,3{ }^{\circ} \mathrm{C}$, obtido pelas ES3, ES4 e ES6.

Os valores de $\mathrm{Tb}$ apresentam diferenças entre espécies, genótipos da mesma espécie e também podem variar em função do estágio de desenvolvimento em que as plantas se encontram (Yang et al.,1995, Lago et al., 2009, Ferreira et al., 2019). Os valores de Tb estimados para a fase de muda do pau-ferro $\left(12,8{ }^{\circ} \mathrm{C}\right)$ e angico-vermelho $(10,3$ ${ }^{\circ} \mathrm{C}$ ) foram próximos a valores encontrados em outros estudos considerando culturas semi-perenes e perenes originárias de locais de clima tropical, como as deste estudo. Como exemplo, a bananeira $\left(10{ }^{\circ} \mathrm{C}\right.$ ) (Robinson et al, 2010), Eucalyptus urophylla $\left(11,5{ }^{\circ} \mathrm{C}\right)$ (Freitas et al., 2017), cultivares de cafeeiro 'Acaiá Cerrado MG-1474' e 'Rubi MG-1192' (12,9 $\left.{ }^{\circ} \mathrm{C}\right)$ (Lima e Silva, 2008), mangueira $\left(10,6{ }^{\circ} \mathrm{C}\right)$ (Callejas et al., 2014), cajueiro-anão Faga-11 $\left(10^{\circ} \mathrm{C}\right)$ (Matos et al., 2014), urucum $\left(12^{\circ} \mathrm{C}\right)$, pau viola $\left(11,4^{\circ} \mathrm{C}\right)$ (Ferreira, et al., 2019) e também para cul-
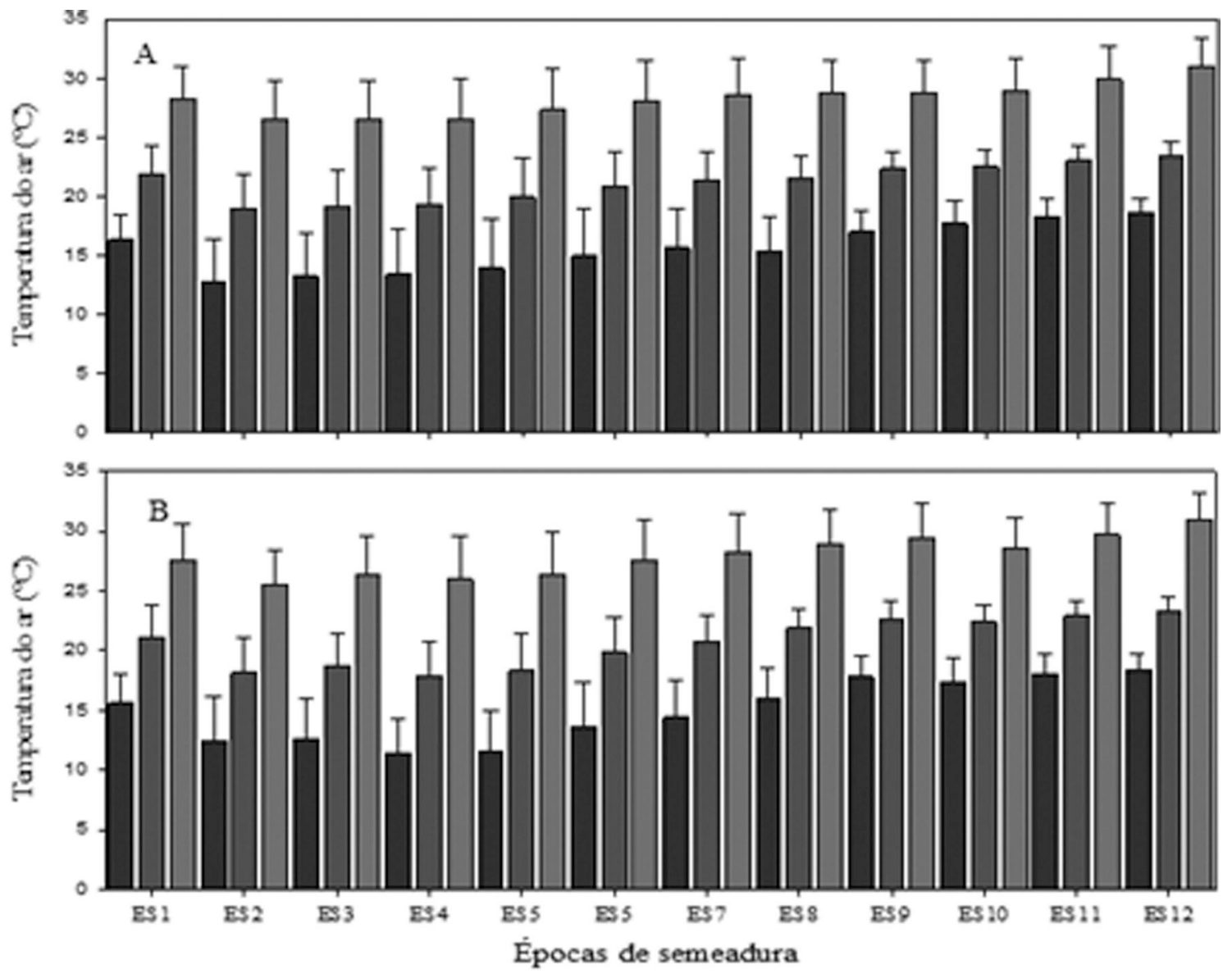

Figura 1 - Caracterização (em ordem) da temperatura mínima, média e máxima do ar ( \pm Desvio Padrão) durante a condução do experimento para o pauferro (A) e angico-vermelho (B). Itajubá, MG, 2017/2018. 
turas perenes de clima temperado como a oliveira cultivar MGSASC $315\left(11,0^{\circ} \mathrm{C}\right)$ (Martins; et al., 2012).

Já a Tot de ambas as espécies foram obtidas através de regressões lineares entre o NF e GD, considerando as Tb's estimadas previamente. As regressões apresentaram coeficiente de ajuste $\left(R^{2}\right)$ elevado $(\geq 0,84$ para o pau-ferro $\mathrm{e} \geq 0,76$ para o angico-vermelho) demonstrando a linearidade entre a emissão de folhas e a temperatura do ar (Figuras 3 e 4). Além disso, indica que a temperatura do ar influencia fortemente o NF em ambas as espécies e a Tot pode ser estimada através da metodologia proposta por Lisboa et al. (2012).

Os valores de filocrono violaram o pressuposto da normalidade pelo teste de Shapiro-Wilk ( $p>0,05)$, sendo necessária a transformação através do $\operatorname{Ln}(\mathrm{x})$, conforme recomendação de Freitas et al. (2017). Os resultados da ANOVA indicaram interação significativa entre as fontes de variação espécie e ES $(\mathrm{p} \leq 0,05)$. Os valores médios de filocrono encontrados para as duas espécies florestais (Tabela 2), representam na prática o DV dado pela quantidade de energia necessária para a emissão de 1 folha na haste principal, e foram de $56,9{ }^{\circ} \mathrm{C}$ dia folha ${ }^{-1}( \pm 17,6)$ para o pau-ferro e $163,8{ }^{\circ} \mathrm{C}$ dia folha ${ }^{-1}( \pm 54,6)$ para o angico-vermelho. Tal diferença mostra que é necessário um acúmulo energético maior no DV do angico-vermelho em relação ao DV do pau-ferro.

Para o pau-ferro, exceto as $\operatorname{ES} 3\left(76,6^{\circ} \mathrm{C}\right.$ dia folha $\left.{ }^{-1}\right)$ e ES9 $\left(101,3{ }^{\circ} \mathrm{C}\right.$ dia folha $\left.{ }^{-1}\right)$, as demais apresentaram menor valor de filocrono e maior DV, sendo as épocas utilizadas para a estimativa da Tot. Para o angico-vermelho as ES utilizadas para a estimativa da Tot foram ES9 $\left(91,6{ }^{\circ} \mathrm{C}\right.$ dia folha $\left.{ }^{-1}\right)$ e $\operatorname{ES} 12\left(85,1^{\circ} \mathrm{C}\right.$ dia folha $\left.{ }^{-1}\right)$, uma vez que apresentaram os menores valores de filocrono e maior DV (Tabela 2). Desta forma, a Tot estimada para o pauferro é de $20,7^{\circ} \mathrm{C}$ e para o angico-vermelho é de $23{ }^{\circ} \mathrm{C}$.
Apesar da diferença entre os valores de filocrono entre ambas as espécies, os valores de Tot foram semelhantes. Visto que o pau-ferro se desenvolveu bem em todas as ES, uma vez que não houve diferença entre os valores de filocrono para a maioria das ES, o valor de Tot é suavizado pela média da Tmed entre as ES. Aliado ao fato de que o angico-vermelho se desenvolveu melhor nas ES9 e ES12, justamente as épocas em que houve maior valor de Tmed, Tmax e Tmin (Figura 1). No entanto, ambos os valores $\left(20,7^{\circ} \mathrm{C}\right.$ e $\left.23^{\circ} \mathrm{C}\right)$ são factíveis sob o ponto de vista biológico e se assemelham às Tot de outras espécies florestais como olho de pavão (Adenanthera pavonina) $\left(24,4{ }^{\circ} \mathrm{C}\right)$, canafístula (Cassia fistula) $\left(24,9^{\circ} \mathrm{C}\right)$, Angelim pedra (Hymenolobium petraeum) $\left(24,9,5^{\circ} \mathrm{C}\right)$ e Angelim saia (Parkia pendula) $\left(25,1^{\circ} \mathrm{C}\right)$ (Monteiro et al., 2014). Cabe ressaltar que os valores de Tot encontrados são próximos às normais climatológicas da região de estudo $\left(21{ }^{\circ} \mathrm{C}\right)$ (Reboita et al., 2015), o que corrobora a ocorrência natural e o bom desenvolvimento das duas espécies na região, além de apresentarem elevado DV durante as épocas de estudo.

As variações da temperatura do ar, tanto diárias quanto sazonais, regulam todas as atividades metabólicas das plantas, uma vez que todos os componentes celulares possuem uma série de termostatos capazes de sentir a temperatura e regular as reações (Bahuguma e Jagadish, 2015). Quanto mais próxima a temperatura do ar estiver da Tot, maior a taxa fotossintética e maior o DV. As espécies tropicais e subtropicais, como as deste estudo, são tipicamente suscetíveis ao dano por resfriamento (Soltani e Sinclair, 2012; Taiz et al., 2018). Isso ocorre quando a temperatura do ar se afasta da Tot em direção à Tb. Nestes casos primeiramente há o enrijecimento da membrana plasmática com consequente perda de função da membrana (Ruelland e Zachowski, 2010). Essa alteração leva à
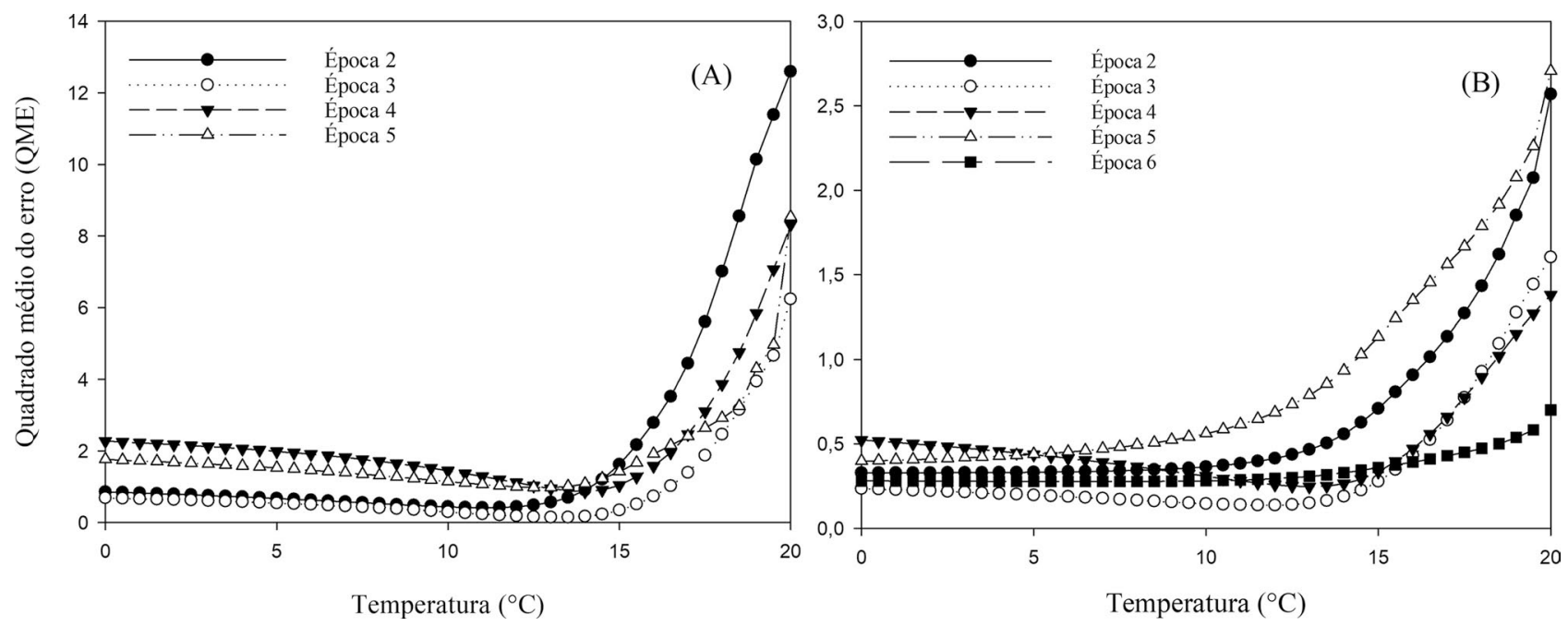

Figura 2 - Quadrado Médio do Erro (QME) da regressão linear simples entre o número de folhas acumuladas na haste principal e os graus-dia acumulado, utilizando valores de temperatura base variando entre $0{ }^{\circ} \mathrm{C}$ a $20{ }^{\circ} \mathrm{C}$, para o pau-ferro (A) e angico-vermelho(B). Itajubá, $\mathrm{MG}, 2017 / 2018$. 

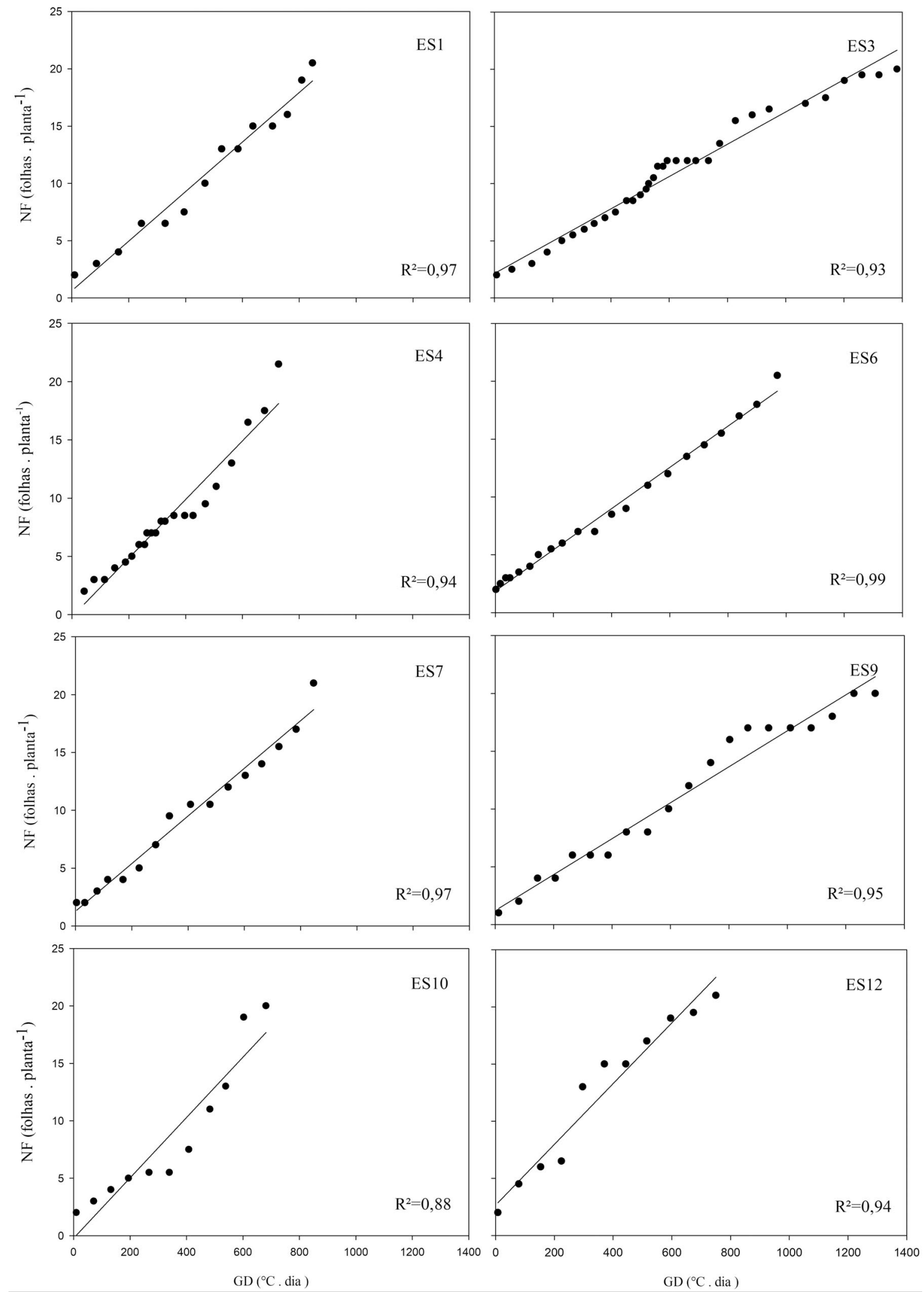

Figura 3 - Relação entre o número de folhas acumuladas na haste principal (NF, folha planta ${ }^{-1}$ ) e os graus-dia (GD, ${ }^{\circ} \mathrm{C}$ dia) acumulado a partir da data de emergência utilizada para a estimativa do filocrono em mudas de pau-ferro. Os dados são representativos de uma unidade experimental em cada época de semeadura - ES1, ES3, ES4, ES6, ES7, ES9, ES10, ES12. Itajubá, MG, 2017/2018. 

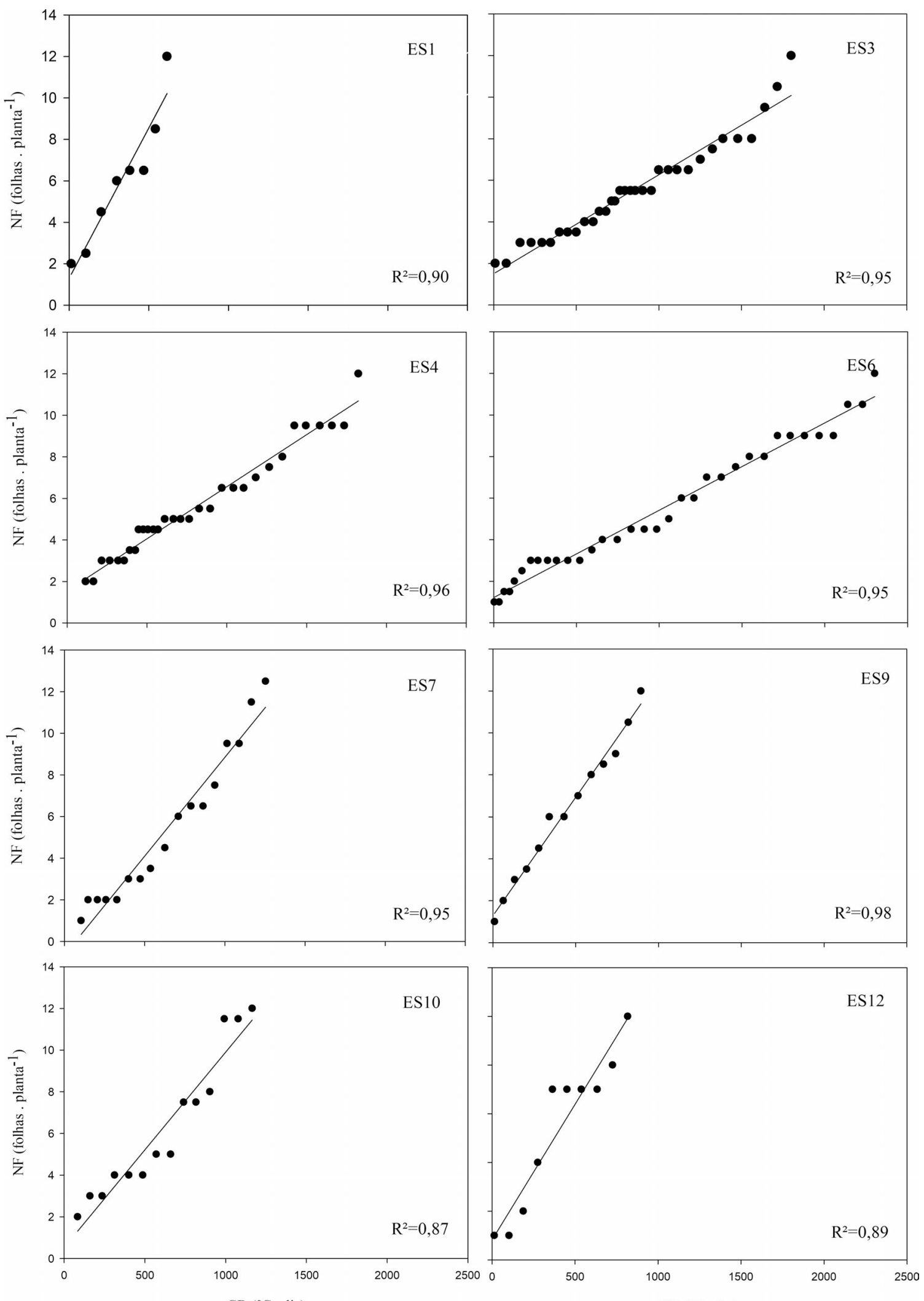

$\mathrm{GD}\left({ }^{\circ} \mathrm{C} \cdot \mathrm{dia}\right)$

$\mathrm{GD}\left({ }^{\circ} \mathrm{C}\right.$. dia $)$

Figura 4 - Relação entre o número de folhas acumuladas na haste principal (NF, folha planta ${ }^{-1}$ ) e os graus-dia (GD, ${ }^{\circ} \mathrm{C}$ dia) acumulado a partir da data de emergência utilizada para a estimativa do filocrono em mudas de angico-vermelho. Os dados são representativo de uma unidade experimental em cada época de semeadura - ES1, ES3, ES4, ES6, ES7, ES9, ES10, ES12. Itajubá, MG, 2017/2018. 
inibição da síntese proteica e aumento da degradação das proteínas existentes, translocação mais lenta de carboidratos, redução das taxas respiratórias e da fotossíntese, além da inibição da atividade hídrica e o estresse osmótico dentro da célula (Ruelland e Zachowski, 2010; Bahuguma e Jagadish, 2015). Todos esses fatores aliados reduzem o DV e consequentemente aumentam a duração da fase de muda nos viveiros florestais (Costa e Streck, 2018; Ferreira et al., 2019) interferindo na comercialização das mesmas.

Neste estudo, o valor estimado de TB de ambas as espécies foi obtido através do Caso I, onde TB $>$ Tmed $>$ Tb. Para o pau-ferro a TB encontrada foi de $46,3{ }^{\circ} \mathrm{C}$, já para o angico-vermelho foi de $45,5{ }^{\circ} \mathrm{C}$, mostrando que ambas são ligeiramente tolerantes a temperaturas mais elevadas, semelhante a outras espécies perenes como o urucum $\left(46,4{ }^{\circ} \mathrm{C}\right)$ (Ferreira et al., 2019), Eucalyptus globulus e Pinus ponderosa $\left(40,0{ }^{\circ} \mathrm{C}\right.$ ) (Sands e Landsberg, 2002; Landsberg et al., 2003), além de Parkia pendula $\left(40,6{ }^{\circ} \mathrm{C}\right)$ (Monteiro et al., 2014), Corymbia citriodora $\left(41,3{ }^{\circ} \mathrm{C}\right)$ e Eucalyptus urophylla $\left(40,5^{\circ} \mathrm{C}\right)$ (Freitas et al., 2017). Valores elevados são esperados, visto que representa o limite máximo de temperatura no qual a planta tem seus processos metabólicos prejudicados (Freitas et al., 2017).

O efeito da temperatura é extremamente complexo e influencia todas as reações bioquímicas da fotossíntese à formação de estruturas moleculares (proteínas, DNA) ou supramoleculares (membranas e cromossomos) (Ruelland e Zachowski, 2010; Soltani e Sinclair, 2012; Bahuguna e Jagadish, 2015). Quando a temperatura $\sim$ da TB pode haver a indução de anomalias no crescimento e desenvolvimento da planta, dentre os quais o abortamento floral, e redução expressiva da taxa fotossintética e redução da emissão de folhas (Soltani e Sinclair, 2012). Tais alterações comprometem o DV, a duração, a qualidade e o estabelecimento dessas mudas no campo. Resumidamente em nível fisiológico quando a temperatura do ar se afasta da Tot em direção à TB há diminuição nas reações da fotossíntese (bruta e líquida) devido a redução na ativação da rubisco - ribulose 1-5 bifosfato carboxilase-oxigenase (Ruelland e Zachowski, 2010), enzima responsável pelo processo metabólico que governa a fotossíntese, e menor afinidade da Rubisco pelo $\mathrm{CO}_{2}$ e maior afinidade pelo $\mathrm{O}_{2}$ com concomitantemente aumento da fotorrespiração (Taiz et al., 2018). Adicionalmente, outras reações e formação de compostos são alteradas (resumidamente) (Ruelland e Zachowski, 2010): aumento da fluidez da membrana, alteração na composição e na estrutura da mesma, reduzindo a estabilidade das membranas devido a redução das ligações de hidrogênio e das interações eletrostáticas das proteínas. Além disso, há o aumento da velocidade das reações enzimáticas, desnaturação das proteínas com consequência na perda de estrutura e da atividade enzimática (Taiz et al., 2018), elevação dos custos de manutenção, devido a fotorrespiração além do comprometimento (derretimento) nas estruturas do RNA (maior detalhamento em Ruelland e Zachowski, 2010).

As TB's obtidas comprovam as características da planta e sua região de origem, as quais são genuinamente de locais tropicais e possuem elevada adaptabilidade em condições de temperatura do ar elevada (Biruel et al., 2010; Dias, 2012). Informações sobre as temperaturas cardinais para espécies arbóreas são escassas em especial para espécies nativas brasileiras, sendo úteis para conhecer as necessidades térmicas e suas adaptações às condições climáticas do local de cultivo (Callejas et al., 2014; Souza e Martins, 2014). Além disso, são variáveis de entrada

Tabela 2 - Comparação de média para o filocrono $\left({ }^{\circ} \mathrm{C}\right.$ dia folha $\left.{ }^{-1}\right)$ para o pau-ferro e angico-vermelho nas doze épocas de semeadura. Itajubá, MG 2017/ 2018 .

\begin{tabular}{lcc}
\hline Época & pau-ferro & angico-vermelho \\
\hline ES1 & $60,8 \mathrm{Aa}$ & $164,2 \mathrm{Bb}$ \\
ES2 & $48,9 \mathrm{Aa}$ & $217,3 \mathrm{Cb}$ \\
ES3 & $76,6 \mathrm{Ba}$ & $225,6 \mathrm{Cb}$ \\
ES4 & $48,7 \mathrm{Aa}$ & $229,2 \mathrm{Cb}$ \\
ES5 & $54,1 \mathrm{Aa}$ & $186,9 \mathrm{Cb}$ \\
ES6 & $62,1 \mathrm{Aa}$ & $234,8 \mathrm{Cb}$ \\
ES7 & $54,6 \mathrm{Aa}$ & $158,6 \mathrm{Cb}$ \\
ES8 & $54,9 \mathrm{Aa}$ & $130,9 \mathrm{Cb}$ \\
ES9 & $101,3 \mathrm{Ba}$ & $91,6 \mathrm{Aa}$ \\
ES10 & $36,9 \mathrm{Aa}$ & $117,6 \mathrm{Bb}$ \\
ES11 & $38,3 \mathrm{Aa}$ & $123,4 \mathrm{Bb}$ \\
ES12 & $45,1 \mathrm{Aa}$ & $85,1 \mathrm{Aa}$ \\
Média global & $56,9 \mathrm{a}$ & $163,8 \mathrm{~b}$
\end{tabular}

Médias seguidas pelas mesmas letras maiúsculas na coluna, para a variável época de semeadura, e minúsculas na linha, para a variável espécie, não diferem entre si pelo teste Scott-Knott $(\alpha=0,05)$. 
importantes nas funções de temperatura dos modelos de desenvolvimento, como o de Wang e Engel, (1998), também utilizados na área florestal e modelos de produtividade florestal, como o 3PG (Sands e Landsberg, 2002; Landsberg et al., 2003; Almeida e Sands, 2016).

\section{Conclusão}

Os valores de temperaturas cardinais, Tb, Tot e TB, respectivamente, para o desenvolvimento vegetativo do pau-ferro (Caesalpinia ferrea) são de $12,8{ }^{\circ} \mathrm{C}, 20,7{ }^{\circ} \mathrm{C}$, $46,3{ }^{\circ} \mathrm{C}$ e para o angico-vermelho (Anadenanthera macrocarpa) são $10,3{ }^{\circ} \mathrm{C}, 23{ }^{\circ} \mathrm{C}, 45,5^{\circ} \mathrm{C}$. As espécies apresentam faixas de temperatura semelhantes sendo consideradas tolerantes a temperaturas mais elevadas. Quanto ao desenvolvimento vegetativo o angico-vermelho é mais lento em relação ao pau-ferro, necessitando de acúmulo maior de energia, em média $163,8^{\circ} \mathrm{C}$ dia folha ${ }^{-1}$, enquanto que o pau-ferro necessita de menor acúmulo, em média $56,9^{\circ} \mathrm{C}$ dia folha ${ }^{-1}$.

\section{Agradecimentos}

À Coordenação de Aperfeiçoamento de Pessoal de Nível Superior (CAPES, processo $\left.n^{\circ} 1671711\right)$ referente à bolsa de estudos do segundo autor e à Fundação de Amparo à Pesquisa de Minas Gerais pelo auxílio financeiro.

\section{Referências}

ABREU, M.C.; MARTINS, F.B.; FREITAS, C.H.; PEREIRA, R.A.A.; MELLONI, E.G. Valores limítrofes para transpiração, desenvolvimento e crescimento de Corymbia citriodora em resposta à deficiência hídrica no solo, Revista Árvore, v. 39, n. 5, p. 841-852, 2015.

ALMEIDA, A.C.; SANDS, P.J. Improving the ability of 3-PG to model the water balance of forests plantations in contrasting environments. Ecohydrology, v. 9, n. 4, p. 610-630, 2016.

ARNOLD, C.Y. The determination and significance of the base temperature in a linear heat unit system. Proceedingsof the American Society for Horticultural Science, v. 74, p. 430-445, 1959.

BAHUGUNA, R.N.; JAGADISH, K.S.V. Temperature regulation of plant phenological development. Environmental Experimental Botany, v. 111, p. 83-90, 2015.

BALDIN, T.; CONTE, B.; DENARDI, L.; MORAES, R.; SALDANHA, C.W. Crescimento de mudas de angico-vermelho em diferentes volumes de Substratos. Pesquisa Florestal Brasileira, v. 35, n. 82, p. 129-133, 2015.

BIRUEL, R.P.; PAULA, R.C.; AGUIAR, I.B. Germinação de sementes de Caesalpinia leiostachya (BENTH.) Ducke (pau-ferro) classificadas pelo tamanho e pela forma. Revista Árvore, v. 34, n. 2, p. 197-204, 2010.

CALLEJAS, I.J.A.; NEVES, G.A.R.; TAVARES, A.S.; MOURA, I.B.; LIMA, E.A. Determinação das temperaturas cardinais da manga cultivar Roxa através de simulação com- putacional utilizando um modelo não linear. Ambiência, v. 10, n. 1, p. 97-110, 2014.

COMISSÃO DE FERTILIDADE DO SOLO DO ESTADO DE MINAS GERAIS. Recomendações para o uso de corretivos e fertilizantes em Minas Gerais: $5^{\circ}$ aproximação. Viçosa: Comissão de Fertilidade do Solo do Estado de Minas Gerais, p. 289-302, 1999.

COSTA, D.B. da; STRECK, N.A. Duração da fase de muda em eucalipto simulada em cenários de aumento de temperatura. Ciência Florestal, n. 28, n. 3, p. 1263-1270, 2018.

DE PAULA, G.M.; STRECK, N.A. Temperatura base para emissão de folhas e nós, filocrono e plastocrono das plantas daninhas papuã e corriola. Ciência Rural, v. 38, n. 9, p. 2457-2463, 2008.

DIAS, P.C.; XAVIER, A.; OLIVEIRA, L.S.; PAIVA, H.N.; CORREIA, C.G. Propagação vegetativa de progênies de meios-irmãos de angico-vermelho (Anadenanthera macrocarpa (Benth) Brenan) por miniestaquia. Revista Árvore, v. 36, n. 3, p. 389-399, 2012.

ERPEN, L.; STRECK, N. A.; UHLMANN, L.O.; LANGNER, J.A.; WINCK, J.E.M.; GABRIEL, L.F. Estimativa das temperaturas cardinais e modelagem do desenvolvimento vegetativo em batata-doce. Revista Brasileira de Engenharia Agrícola e Ambiental, v. 17, n. 11, p. 1230-1238, 2013.

FAGUNDES, J.D.; STRECK, N.A.; STORCK, L.; REINIGER, L.R. Temperatura base e soma térmica de subperíodos de desenvolvimento de Aspilia montevidensis. Bragantia, v. 69, n. 9, p. 499-507, 2010.

FERREIRA, D.F. Sisvar: a Guide for its Bootstrap procedures in multiple comparisons. Ciência e Agrotecnologia, v. 38, n. 2, p. 109-112, 2014.

FERREIRA, M.C.; MARTINS. F.B.; FLORÊNCIO, G.W.L.; SILVA, J.P.C.; PASIN, L.A.A.P. Cardinal temperatures and thermal requirements for initial development of two Brazilian native species. Pesquisa Agropecuária Brasileira, v. 54, e00525, 2019.

FREITAS, C.H.; MARTINS, F.B.; ABREU, M.C. Cardinal temperatures for the leaf development of Corymbia citriodora and Eucalyptus urophylla seedlings. Pesquisa Agropecuária Brasileira, v. 52, n. 5, p. 283-292, 2017.

GARCÍA-MOZO, H.; MESTRE, A. GALÁN, C. Phenological trends in southern Spain: A response to climate change. Agricultural and Forest Meteorology, v. 150, p. 575-580, 2010.

GARCIA, S. R.; SANTOS, D. F.; MARTINS, F. B.; TORRES, R. R. Aspectos climatológicos associados ao cultivo da oliveira (Olea europaea L.) em Minas Gerais. Revista Brasileira de Climatologia, v. 22, p. 188-209, 2018.

LAGO, I.; STRECK, N.A.; CARVALHO, M.P.; FAGUNDES, L.K.; DE PAULA, G.M.; LOPES, S.J. Estimativa da temperatura base do subperíodo emergência-diferenciação da panícula em arroz cultivado e arroz vermelho. Revista Ceres, v. 56, n. 3, p. 288-295, 2009.

LANDSBERG, J.J.; WARING, R.H.; COOPS, N.C. Performance of the forest productivity model 3-PG applied to a wide range of forests types. Forest Ecology and Management, v. 172, n. 2-3, p. 199-214, 2003.

LIMA, E.P.; SILVA, E.L. Temperatura base, coeficientes de cultura e graus-dia para cafeeiro arábico em fase de implanta- 
ção Revista Brasileira de Engenharia Agrícola e Ambiental, v. 12, n. 3, p. 266-273, 2008.

LISBOA, P.M.M.; MARTINS, F.B.; ALVARENGA, M.I.N.; NETO, J.V.; REIS, D.F. Desenvolvimento vegetativo de duas cultivares de oliveira na fase de muda. Ciência Rural, v. 42, n. 9, p. 1556-1562, 2012.

LUCAS, D.D.P.; STRECK, N.A.; BORTOLUZZI, M.P.; TRENTIN, R.; MALDANER, I. Temperatura base para emissão de nós e plastocrono de plantas de melancia. Revista Ciência Agronômica, v. 43, n. 2, p. 288-292, 2012.

MARENCO, R.A.; LOPES, N.F. Fisiologia vegetal: fotossíntese, respiração, relações hídricas e nutrição mineral. 3 . ed. Viçosa: UFV, 2009. 486 p.

MARTINS, F.B.; FERREIRA, M.C.de; OLIVEIRA, L.F.S.dos; RAMALHO, V.; GONÇALVES, E.D. Temperatura do ar no desenvolvimento reprodutivo de cultivares de oliveira. Revista Brasileira de Meteorologia, v. 34, n. 2, p. 179190, 2019.

MARTINS, F.B.; GONZAGA, G.; SANTOS, D.F. dos; REBOITA, M.S. Classificação climática de Köppen e de Thornthwaite para Minas Gerais: cenário atual e projeções futuras. Revista Brasileira de Climatologia, v. 14, edição especial dossiê climatologia de Minas Gerais, p. 129-156, 2018.

MARTINS, F.B.; REIS, D.F.; PINHEIRO, M.V.M. Temperatura base e filocrono em duas cultivares de oliveira. Ciência Rural, v. 42, n. 11, p. 1975-1981, 2012.

MARTINS, F.B.; SILVA, J.C.; STRECK, N.A. Estimativa da temperatura-base para emissão de folhas e do filocrono em duas espécies de eucalipto na fase de muda. Revista Árvore, v. 31 n. 3, p. 373-381, 2007.

MATOS, V.A.T.; PIVETTA, F.; PAIVA SOBRINHO, S.; TISSIANI, A. S.O.; PEREIRA, A.P.M.S.; RAMOS, F.T.; CAMPELO JÚNIOR, J.H. Temperaturas basais e exigência térmica para a maturação de caju. Bioscience Journal, v. 30, n. 4, p. 969-977, 2014.

MIZUTANI, M.; KANAOKA, M.M. Environmental sensing and morphological plasticity in plants. Seminars in Cell $\&$ Developmental Biology, v. 83, p. 69-77, 2018.

MONTEIRO, E.B.; DA SILVA, C.C.; DA SILVA, A.C.; SOUZA, A.P. de. Estimating emission of leaves seedings Forest in different shading levels, at conditions of transition Amazon-Cerrado, Brazil. American Journal of Plant Sciences, v. 5, n. 15, p. 2330-2341, 2014.

MUTTONI, M.; ALBERTO, C.M.; BARTZ, A.C.; UHLMAN, L.O. ; TARTAGLIA, V.L.; STRECK, N.A. Cardinal temperatures for planting-emergence phase in gladiolus. Ciência Rural, v. 47, n. 10, e20160824, 2017.

OLIVEIRA, M.C. de; OGATA, R.S.; ANDRADE, G.A.de; SANTOS, D.S.; SOUZA, R.M.; GUIMARÃES, T.G.; SILVA JUNIOR, M.C.da; PEREIRA, D.J.S.; RIBEIRO, J.F. Manual de viveiro e produção de mudas: espécies arbóreas nativas do cerrado. Editora rede de sementes do cerrado: Brasília, 2016. 124 p.
OMETTO, J.C. Bioclimatologia Vegetal. São Paulo: ed. Agronômica Ceres, 440 p., 1981.

REBOITA, M.S.; RODRIGUES, M.; SILVA, L.F.; ALVES, M.A. Aspectos climáticos do Estado de Minas Gerais. Revista Brasileira de Climatologia, v. 17, p. 206-226, 2015.

ROBINSON, J.C.; GALÁN SAÚCO, V. Bananas and plantains. 2 ed. Oxford: CAB International. 2010. 311p. (Crop production science in horticulturae series, 19).

RUELLAND, E.; ZACHOWSKI, A. How plants sense temperature. Environmental Experimental Botany, v. 69, p. 225232, 2010.

SANDS, P.J.; LANDSBERG, J.J. Parameterizationof 3-PG for plantation grown Eucalyptus globulus. Forest Ecology and Management, v. 163, p. 273-292, 2002.

SCOTT, A.J.; KNOTT, M. A cluster analysis method for grouping means in the analysis of variance. Biometrics, v. 30, n. 3, p. 507-512, 1974.

SINCLAIR, T.R.; GILBERT, R.A.; PERDOMO, R.E.; SHINE, J.M.; POWELL, G.; MONTES, G. Sugarcane leaf area development under field conditions in Florida, USA. Field Crops Research, v. 88, n. 1, p. 171-178, 2004.

SOLTANI, A.; SINCLAIR, T.R. Modeling Physiology of crop development, growth and yield. Oxfordshire: CAB Internacional, 2012, $322 \mathrm{p}$.

SOUZA, P.M.B.; MARTINS, F.B. Estimativa da temperatura basal inferior para as cultivares de oliveira Grappolo e Maria da Fé. Revista Brasileira de Meteorologia, v. 29, n. 2, p. 307-313, 2014.

STRECK, N.A.; PAULA F.L.M.; BISOGNIN D.A.; HELDWEIN A.B.; DELLAI, J. Simulating the development of field grown potato (Solanum tuberosum L.). Agricultural and Forest Meteorology,v. 1 42, n. 1, p. 1-11, 2007.

STRECK, N.A.; LAGO, I.; SAMBORANHA, F.K.; GABRIEL, L.F.; SCHWANTES, A.P.; SCHONS, A. Temperatura base para aparecimento de folhas e filocrono da variedade de milho BRS Missões. Ciência Rural, v. 39, n. 1, p. 224-227, 2009.

STRECK, N.A.; UHLMANN, L.O.; GABRIEL, L.F. Leaf development of cultivated rice and weedy red rice under elevated temperature scenarios. Revista Brasileira de Engenharia Agrícola e Ambiental, v. 17, n. 2, p. 190-199, 2013.

TAIZ, L; ZEIGER, E.; MOLLER, I.M.; MURPHY, A. Plant Physiology and Development. $6^{\mathrm{a}}$ ed. Oxford: Oxford University Press, 2018. 513p.

YANG S.; LOGAN, J.; COFFEY, D.L. Mathematical formulae for calculating the base temperature for growing degree days. Agricultural and Forest Meteorology, v. 75, p. 6174, 1995.

License information: This is an open-access article distributed under the terms of the Creative Commons Attribution License (type CC-BY), which permits unrestricted use, distribution and reproduction in any medium, provided the original article is properly cited. 